\title{
DIFFÉRENTS NIVEAUX D'ANALYSE DU CONTEXTE DANS L'ÉTUDE ERGONOMIQUE DU TRAVAIL COLLECTIF*
}

\author{
Laurent Karsenty ${ }^{l}$ et Bernard Pavard
}

\section{Introduction}

Nous concevons le travail collectif comme l'articulation de compétences et/ou de tâches individuelles nécessaire à l'accomplissement d'un but commun. Cette articulation ne va pas toujours de soi : elle suppose elle-même un certain travail (Schmidt, 1994). Le travail d'articulation peut reposer sur des mécanismes de coordination préétablis : le kanban, dispositif inventé au Japon pour faciliter la gestion de stocks, est un exemple de mécanisme de coordination (voir Schmidt \& Simone, 1995). Cependant, dans nombre de situations de travail, de tels mécanismes de coordination n'existent pas. Le travail d'articulation repose alors de façon essentielle sur la communication et l'exploitation d'un ensemble de ressources contextuelles.

Nous aurons l'occasion d'illustrer ce constat tout au long de ce texte par différentes études. Il nous conduit à mettre au centre de l'étude du travail collectif la notion de contexte. Nous définissons le contexte comme l'ensemble des ressources contingentes à l'activité des opérateurs exploitées pour donner sens à la situation de travail et y répondre de la façon la plus adéquate possible. Ces ressources sont soit internes à chaque opérateur - contenus des mémoires perceptives, de la mémoire de travail ou de la mémoire à long-terme (ces concepts sont repris et définis dans la suite) - soit externes - environnement de travail, comportements des collègues, règles organisationnelles, etc.

La notion de contexte avancée dans cet article a une autre caractéristique importante liée aux données utilisées pour contextualiser une information à traiter : leur prise en compte par l'opérateur dépend d'une activité opportuniste. Si l'opérateur a un besoin d'information à un moment donné (pour désambiguïser une information, pour comprendre la cause de l'état décrit, pour choisir la meilleure décision, etc.), il prélève "au vol" des données utiles qui lui sont accessibles. Il est ainsi possible de rendre compte du fait que le contexte peut prendre tant de formes différentes, et qu'il est souvent difficile de prévoir quelles seront les données contextuelles qui seront exploitées, et comment elles le seront (voir figure 1).

On peut rattacher à cette position le fait que l'exploitation des données contextuelles n'est le plus souvent pas prévue dans la définition des tâches des opérateurs, ni dans la définition de leur environnement de travail. De même, les opérateurs ne sont pas toujours conscients de les avoir utilisées, ce qui se traduit par une difficulté voire une incapacité à en rendre compte lors d'entretiens d'explicitation ou de recueil d'expertise. Cette incapacité des opérateurs peut s'expliquer de deux façons : (1) l'exploitation du contexte relève de processus

\footnotetext{
* Texte paru en 1997 dans la revue Réseaux, n85, pp.73-99.

${ }^{1}$ Contact : laurent.karsenty@laposte.net
} 
ne nécessitant pas un contrôle conscient (processus sub-attentionnels) ; (2) le contexte utilisé pour prendre une décision, du fait de son caractère opportuniste, ne relève pas d'un savoirfaire formalisé, donc appris ou susceptible de l'être ; de ce fait, la mémorisation des données contextuelles est plus aléatoire.

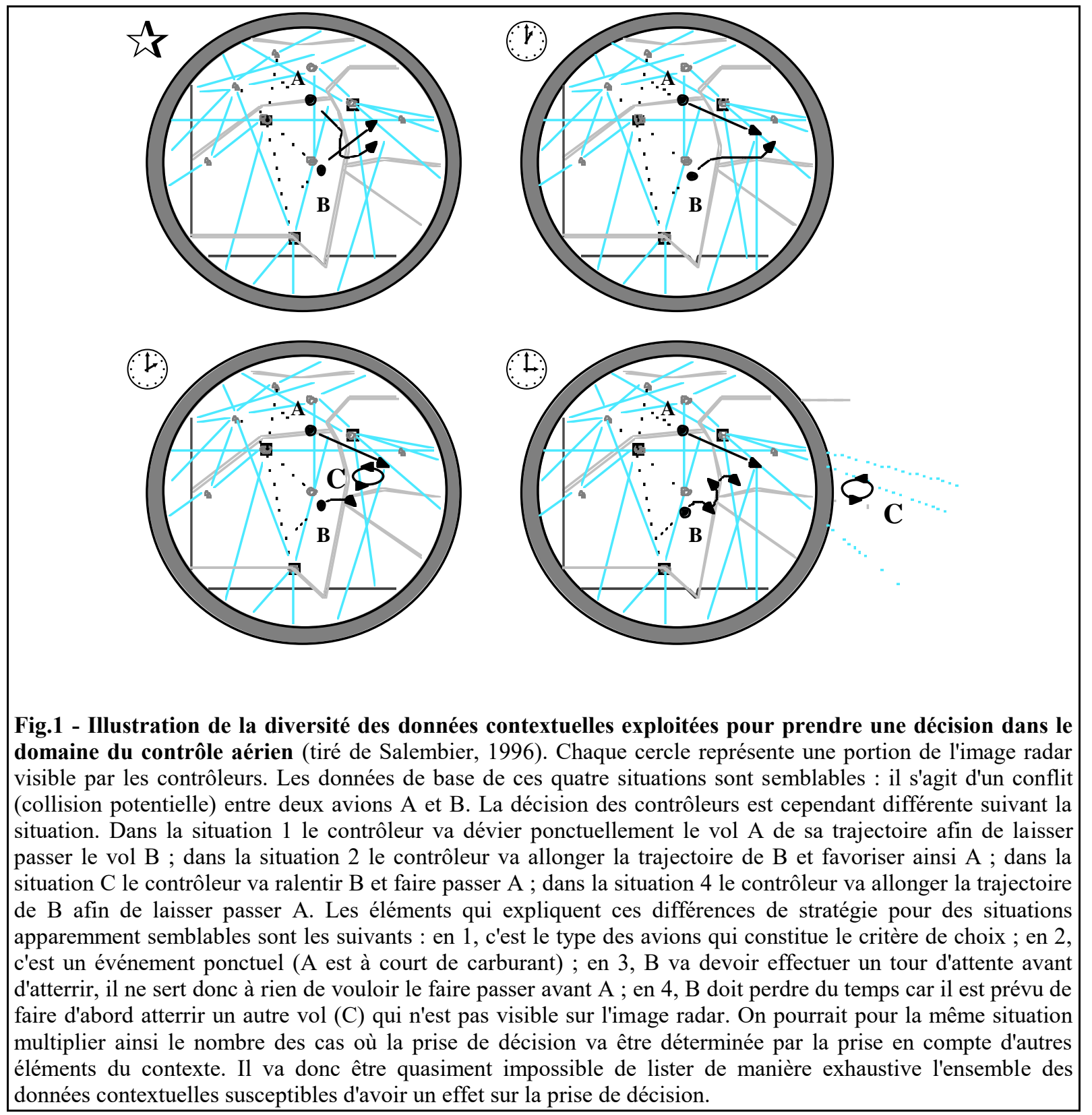

Le caractère opportuniste de la constitution du contexte est vraisemblablement l'une des raisons qui explique pourquoi la notion de contexte n'a été introduite que relativement tardivement dans l'étude de la cognition individuelle et sociale. Il est vrai que le contexte a de ce fait un caractère polysémique et ubiquitaire, ce qui a pu conduire certains psychologues à n'y voir qu'un résidu d'ignorance, voire une véritable "poubelle conceptuelle" (Smith, Glenneberg \& Bjork, 1978 cité dans Hoffman, 1986). Il n'en reste pas moins que l'importance théorique du concept de contexte est aujourd'hui largement reconnue en sciences cognitives, que ce soit dans le champ des théories de la perception (ex., Gibson, 1979) de l'apprentissage (ex., Hoffman, 1986), de la mémoire (ex., Davies, 1986), de la communication (ex. Sperber \& 
Wilson, 1986) de l'interaction homme-machine (ex., Suchman, 1987, Winograd \& Florès, 1987) et de l'intelligence artificielle (ex., Brézillon \& Cavalcanti, 1997).

En ce qui concerne l'ergonomie, qui est notre discipline spécifique, le concept de contexte n'est devenu un thème central d'étude que depuis quelques années, et ce même si nombre d'ergonomes francophones faisaient et font encore, sans le savoir forcément, une étude approfondie du contexte lorsqu'ils interviennent dans la conception ou l'évaluation d'une situation de travail. L'analyse du contexte est en effet inhérente à l'un des fondements de l'ergonomie francophone : la distinction entre tâche et activité (Leplat et Hoc, 1983) : "La tâche, c'est le but à atteindre et les conditions dans lesquelles il doit être atteint. L'activité, c'est ce qui est mis en œuvre par le sujet pour exécuter la tâche" (Leplat, 1986, p.10). La tâche représente donc l'aspect prescrit du travail, l'activité son aspect effectif. La spécificité de l'analyse ergonomique d'une situation de travail est d'appliquer des méthodes particulières pour déceler les décalages entre la tâche et l'activité réelle des opérateurs : ces décalages peuvent être signes de dysfonctionnement ou d'inadaptation de la définition de la tâche, et leur identification peut permettre d'envisager des actions régulatrices. En fait, la possibilité de prescrire des actions régulatrices suppose de comprendre pourquoi l'activité ne se conforme pas à la tâche prescrite. C'est dans cette tentative pour comprendre les décalages entre tâche et activité que l'ergonome est amené à faire une étude approfondie du contexte. Nous citerons quelques exemples issus des travaux menés par les membres de notre laboratoire :

L'analyse de l'activité d'exécution de check-lists par des pilotes a montré que ceux-ci exploitaient leur connaissance de la situation de vol, du fonctionnement de l'avion et de procédures exécutées dans le passé pour décider si la procédure imposée par la compagnie était adéquate ou non (Karsenty, Bigot \& De Brito, 1995). Cette caractéristique de l'activité des pilotes est en contradiction avec la tâche qui leur est assignée, en particulier dans des situations de panne, qui stipule que les pilotes doivent se contenter d'appliquer à la lettre et immédiatement les procédures prévues par le constructeur et la compagnie.

- L'analyse de l'activité de conduite de réacteur nucléaire a révélé que les prises de décision individuelles reposaient sur d'autres informations que celles prévues au niveau de chaque poste de travail. Les opérateurs en salle exploitent notamment des messages pris "au vol" émis par un interphone disposé dans la salle de contrôle, et diffusant les communications entre le chef de salle et des techniciens situés à proximité du réacteur (Rognin \& Pavard, 1996).

_ L'étude de l'activité dans un centre SAMU (urgences médicales) a montré que les mécanismes collectifs de régulation visant à répondre le plus vite et le plus efficacement aux appels téléphoniques, reposent sur la perception de la disponibilité des autres partenaires (Pavard, Benchekroun, \& Salembier, 1990). Cette perception de la disponibilité d'autrui suppose de pouvoir analyser les postures d'autrui et de disposer d'une connaissance des appels en cours de traitement, ainsi que de leur niveau d'urgence. Ceci est permis parce que les opérateurs du centre SAMU, deux médecins et deux permanencières, sont présents dans la même salle et peuvent se voir et entendre les communications téléphoniques de chacun.

En somme, l'analyse du contexte est partie intégrante de l'analyse de l'activité. L'ergonomie intègre ainsi la notion de contexte dans sa démarche même. Mais cette notion n'a pas toujours été identifiée comme telle (à quelques exceptions près, notamment Terssac \& Chabaud, 1990), et reste difficile à cerner. Nous pensons que la clarification de la notion de contexte peut aider à mieux orienter l'analyse ergonomique de l'activité. L'ergonome dirigera son attention plus rapidement sur des aspects pertinents de l'activité, et, si nécessaire, aura la possibilité d'aboutir à une description complète des dimensions contextuelles de l'activité. En 
outre, lorsqu'il s'agit d'activités collectives ou de communications de travail, la prise en compte du contexte devient cruciale dans la mesure où l'intelligibilité mutuelle des actions de chacun repose sur le partage du contexte interprétatif (Sperber \& Wilson, 1986, Schmidt \& Bannon, 1992, Karsenty \& Falzon, 1993). Aider des agents à communiquer et à se comprendre peut passer par une aide à la contextualisation. La définition d'une telle aide suppose une analyse fine du contexte de la coopération. Enfin, d'un point de vue théorique, l'analyse de la cognition et de la communication en contexte entretient un rapport particulier avec les sciences expérimentales, qui procèdent en décontextualisant volontairement chaque processus étudié pour en établir les lois internes : elle peut d'un côté conduire à valider les modèles ainsi établis, d'un autre pousser à une extension de ces modèles (ex., Hutchins, 1990, Hutchins, 1995, Zhang \& Norman, 1994), voire carrément à une reformulation (ex., Suchman, 1987).

L'objet de cet article est de proposer le résultat de quelques réflexions visant à clarifier la notion de contexte. Différents niveaux d'intervention de la notion de contexte dans l'analyse ergonomique des situations de travail seront proposés et illustrés par des études centrées sur chaque niveau. Des conséquences méthodologiques de l'intervention de ces différents niveaux de contexte seront identifiées dans la conclusion.

\section{Cadre général}

Il est possible de distinguer différents niveaux ou types de contexte. Certaines études en psychologie cognitive distinguent ainsi le contexte interne (état émotionnel, besoins) du contexte externe (Davies, 1986), ou encore le contexte situationnel (ensemble des stimuli physiquement proches du stimulus focal), et le contexte représentationnel, (ensemble des représentations mentales présentes en mémoire de travail, Tiberghien, 1985). Ces distinctions, bien qu'utiles dans un premier temps, sont insuffisantes pour prendre en compte toute la complexité des situations de travail. Elles s'avèrent en outre trop grossières pour nos objectifs.

La pragmatique linguistique et l'ethnographie, qui abordent l'étude du langage à travers son utilisation, ont mis l'accent très tôt sur l'importance du contexte dans l'interprétation des énoncés. Plusieurs dimensions du contexte ont été proposées (ex., Brown \& Yule, 1983, chap.2) : le temps et l'endroit de l'interaction, le locuteur, l'auditeur, le thème de la discussion, le discours précédent, la forme de la conversation (débat, conversation libre, sermon, etc.), l'objectif du dialogue. Tout en reconnaissant l'apport de ces travaux, nous considérons qu'ils véhiculent une conception en partie erronée du contexte lorsque l'on veut étudier l'interaction humaine : il n'existe pas un temps et un lieu de l'interaction, ni $u n$ locuteur et $u n$ auditeur, ni même un thème de discussion indépendamment des acteurs en présence. Chaque acteur donne un sens particulier à la situation dans laquelle l'interaction prend place. Invoquer l'importance de l'auditeur, par exemple, pour comprendre comment est formulé un énoncé est pertinent, mais ce n'est pas en soi l'auditeur qui sera responsable du contenu et de la forme de l'énoncé, c'est la représentation qu'en a le locuteur (s'il le considère comme un supérieur hiérarchique ou d'un statut symétrique, s'il le considère comme quelqu'un de compétent ou non sur un domaine de discours donné, etc.). Nous considérons par conséquent que le contexte doit être appréhendé d'un point de vue subjectif si l'on veut comprendre l'activité : l'analyste ne peut statuer a priori sur la définition d'une situation; il doit chercher quelle est la représentation de la situation pour chaque opérateur. Ainsi, l'existence d'objets ou d'événements particuliers ne peut pas être considérée en soi comme suffisante pour décrire le contexte d'une activité ; il est nécessaire de déterminer quels objets et événements sont accessibles à l'opérateur, et quel sens ils ont pour lui.

En partant de ces considérations, et en prenant spécifiquement en compte les dialogues de travail, Cahour \& Karsenty (1996) ont proposé une classification des contextes cognitifs. 
Cette classification inclut : (1) le contexte du dialogue : mémoire des échanges passés, type de dialogue (ex., conversation formelle ou informelle), (2) le contexte de la tâche : buts poursuivis, étapes de la tâche déjà réalisées, contraintes à satisfaire, (3) le contexte de l'environnement externe, dans ses dimensions spatiales (localisation des objets) et temporelles, (4) les représentations psychosociales associées aux interlocuteurs : statuts, compétences, préférences, etc. (5) le contexte supposé partagé entre les interlocuteurs, qui contient des éléments présents au niveau individuel dans chacun des quatre autres contextes. La notion de contexte supposé partagé était préférée à celle de contexte partagé, car cette dernière peut présupposer l'existence d'un ensemble de croyances objectivement partagées, et donc pouvant être considérées comme telles par un analyste extérieur. Or l'analyse des dysfonctionnements de la communication (incompréhension, malentendu, quiproquo) montre justement que l'erreur vient souvent d'un fait supposé à tort partagé, et donc laissé implicite par le locuteur. D'un point de vue psychologique, il est donc plus juste d'étudier les contextes supposés partagés par les membres d'un collectif que le contexte partagé du collectif. Enfin, les auteurs soulignaient l'existence de contextes privés, c'est-à-dire de croyances sur le dialogue, la tâche, l'environnement ou l'interlocuteur dont le détenteur sait consciemment qu'elles ne sont pas partagées.

Cette classification est directement pertinente pour traiter des dialogues de travail, mais plus particulièrement des dialogues en face-à-face, à composante essentiellement verbale. Elle s'avère incomplète pour traiter de situations de travail plus complexes, et ce pour trois raisons

la communication est multi-modale : les interlocuteurs peuvent utiliser des modes de communication non verbaux (gestes, expressions faciales, etc.), conjointement ou non au verbal, ainsi que des modes de communication médiatisés par des artefacts ;

- le rôle des artefacts dans la communication et la coordination conduit à mettre l'accent sur un autre niveau de contexte, le niveau perceptif ;

enfin, d'autres contextes sont à prendre en compte pour aborder l'étude de collectifs plus grands, notamment la structure organisationnelle du travail et la culture des opérateurs. 


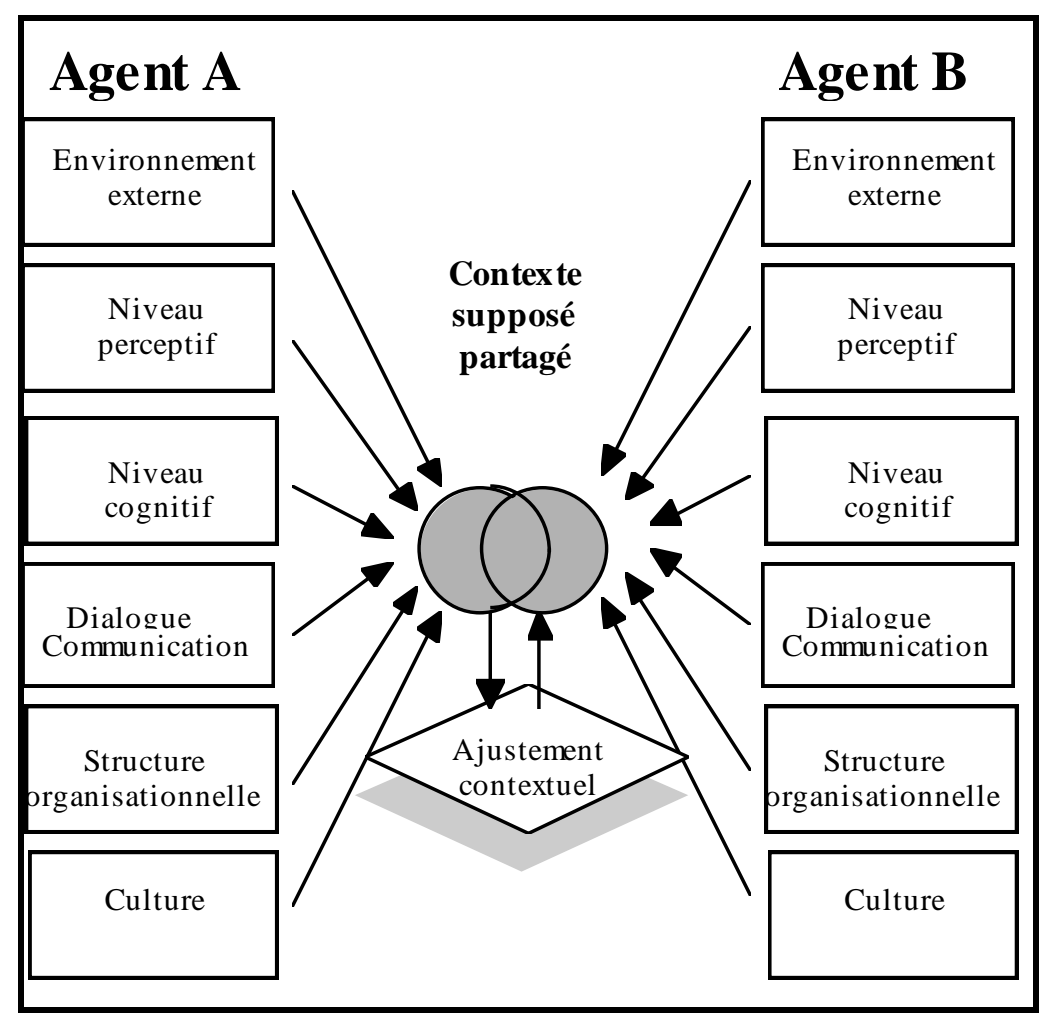

Fig. 2 - Les niveaux de contexte pouvant être employés pour interpréter l'activité dans les situations de travail. Le contexte peut intervenir soit au niveau individuel soit au niveau collectif, notamment dans les situations de coopération (besoin de partager le même contexte)

Les niveaux de contexte caractérisant une situation de travail complexe incluent donc d'autres dimensions que celles qui sont présentes dans le dialogue en face-à-face et à deux. La figure 2 représente un cadre général incluant ces différents niveaux de contexte. On représente dans cette figure le fait qu'à chaque moment de l'activité, chaque agent exploite, consciemment ou parfois inconsciemment, un ou plusieurs contenus issus des différents types de contexte (représentés dans la figure 2 par des boîtes). La compréhension mutuelle des actions de chacun et la coordination suppose un certain degré de contexte partagé (l'intersection entre les contextes individuels).

La figure 2 représente aussi le fait que le contexte, en particulier le contexte interprétatif, n'est pas imposé aux agents : il peut être le résultat d'une série d'ajustements, le produit d'une véritable négociation.

\section{Différents niveaux d'analyse du contexte}

\section{L'environnement externe}

L'ergonomie a depuis longtemps mis l'accent sur la nécessité d'étudier l'environnement de travail pour comprendre l'activité. L'étude de l'environnement de travail a notamment été abordée du point de vue des différentes sources de nuisance (chaleur, luminosité, bruit, vibrations, etc.), de la configuration physique du poste de travail, de la lisibilité des informations utiles aux opérateurs, ou encore des aides au travail disponibles (procédures, modes d'emploi, etc.). 
Toutes ces dimensions ne sont pas directement liées à la problématique du contexte telle qu'elle a été énoncée dans cet article : tous les aspects de l'environnement de travail ne fournissent pas les ressources nécessaires aux opérateurs pour donner du sens à la situation de travail et y répondre le plus adéquatement. Toutefois, nous considérons que la problématique du contexte énoncée ici est un prolongement de ce souci constant de l'ergonomie pour comprendre l'activité de l'opérateur dans sa relation avec son environnement.

$\mathrm{Du}$ point de vue de l'analyse du contexte, on peut distinguer deux rôles de l'environnement externe.

1. L'environnement externe fournit des données qui peuvent être utilisées pour contextualiser une information à traiter. Les études sur l'utilisation de supports externes dans les réunions de travail (ex., tableaux, feuilles de dessin, etc.) illustrent cette fonction de l'environnement externe. Tang (1991) par exemple, étudiant des réunions de conception en petits groupe ( 3 ou 4 personnes), observe qu'un croquis réalisé sur une feuille de papier fournit le contexte nécessaire pour comprendre les gestes de la main des participants à la réunion. Un autre exemple est donné par les travaux visant à doter les projets de conception d'une nouvelle documentation, informatisée ou non, décrivant le contexte de la conception (ex., Conklin \& Begeman, 1988, MacLean et al., 1991, Karsenty, 1996a). Le contexte de la conception est conçu ici comme une structure argumentative co-construite par les membres d'un projet, composée des problèmes posés, des solutions alternatives envisagées par le groupe pour résoudre ces différents problèmes, et des arguments pour ou contre chaque solution alternative. Cette structure argumentative peut être dotée d'une dimension temporelle, dans la mesure où l'on représente l'enchaînement des problèmes et des solutions discutés au cours d'un projet. Elle est par ailleurs un reflet du raisonnement situé des concepteurs, dans la mesure où interviennent dans les arguments des uns et des autres des contraintes très spécifiques de chaque projet (tel spécialiste n'étant pas disponible, on ne pouvait pas avoir l'estimation nécessaire pour choisir entre les solutions $\mathrm{X}$ et $\mathrm{Y}$; telle technologie était plus performante, mais trop chère par rapport au budget alloué au projet, etc.). Le besoin d'une documentation décrivant le contexte de la conception s'est notamment fait sentir dans les projets où l'on doit réutiliser une solution passée - cette solution peut alors être envisagée dans un contexte d'alternatives et dans un contexte de critères de conception particuliers au projet passé - ou pour faciliter l'introduction de nouveaux acteurs dans des grands projets, généralement très étalés dans le temps (cf. Conklin \& Yakemovic, 1991).

2. L'environnement externe intègre des contraintes qui sélectionnent l'ensemble des interprétations et des décisions d'action possibles. Zhang \& Norman (1994) ont développé une théorie et une méthodologie permettant de prendre en compte le fait que pour la plupart des tâches effectuées en situation naturelle, les objets qui nous entourent jouent implicitement un rôle important dans l'organisation de la cognition. Selon cette approche, les représentations externes interviennent en temps que contraintes ou règles implicites. Par exemple, un raisonnement sur la dynamique d'un processus continu (ex. maintenir la température d'une pièce) va dépendre du type de représentation utilisé pour visualiser les informations : une représentation digitale des paramètres à contrôler (la température par exemple) induira un raisonnement très différent de celui issu d'une représentation analogique, qui elle permettra de visualiser des tendances, de raisonner de façon qualitative, et de mieux anticiper l'évolution des variables. L'apport de Zhang et Norman est d'avoir développé un cadre théorique et méthodologique (l'analyse représentationnelle) permettant une description systématique du rôle des composantes externes et internes des systèmes représentationnels intervenant dans les processus cognitifs. Pour chaque niveau de la hiérarchie, la méthodologie impose d'identifier ses 
propriétés représentationnelles. Notons au passage que l'hypothèse d'analyse par décomposition hiérarchique limite considérablement la portée de l'approche. En effet, pour la plupart des tâches coopératives, les activités des acteurs ne peuvent se décomposer de façon hiérarchique, mais résultent de processus opportunistes (lesquels sont néanmoins contraints par les propriétés représentationnelles des objets externes à l'opérateur comme le rappellent Zhang et Norman). En conclusion, les notions d'objet et de représentation externe jouent ici le rôle de contexte pour la cognition dans la mesure où chaque agent opérant dans un environnement complexe prend implicitement (inconsciemment) en compte les contraintes associées aux objets qui l'entourent. En ce sens, les travaux de Zhang et Norman gardent une valeur opérationnelle importante car ils nous forcent à analyser systématiquement, pour chaque type d'activité (et non de tâches) le rôle représentationnel que peut jouer chaque objet de l'environnement (voir Zorola, Pavard \& Bastide, 1997 pour une formalisation du rôle représentationnel des artefacts en situation de coopération).

\section{Le niveau perceptif}

Dans quelle mesure les mécanismes de perception de l'information sont-ils dépendant ou indépendant du contexte dans lequel se situe le sujet ? Notre objectif n'est évidemment pas de faire une revue de question de cette problématique classique en psychologie cognitive car elle a été et reste l'objet d'une part importante des recherches menées dans ce champ.

Nous rappellerons simplement que deux écoles se confrontent : (1) celle des tenants de processus où la perception s'effectue dans un premier temps à partir de modules autonomes, indépendant du contexte fourni par les processus de niveau supérieur, et (2) celle où le contexte peut directement influencer les opérations les plus élémentaires de la perception, comme la reconnaissance d'une forme ou l'attribution du sens à un mot entendu ou lu. Cela dit, si l'on s'intéresse non pas aux processus de pré-traitement de l'information mais au résultat de cette activité, tout le monde s'accorde à reconnaître que le contexte cognitif dans lequel se situe l'activité perceptive joue un rôle déterminant.

Dans une série d'expériences qui avait pour thème l'analyse des stratégies de lecture chez les professionnels (Pavard, 1983), nous avons pu mettre en évidence le rôle du contexte sur les processus d'accès au lexique (c'est-à-dire le processus par lequel les mots sont progressivement transformés de graphèmes en unités de sens). Plusieurs variables externes (i.e., liées à la structure du document) écrit ont été analysées : la structure morphologique des mots, la structure syntaxique de la phrase, ainsi que des variables internes (i.e., liées à l'état cognitif du lecteur) : niveau de compréhension du texte, objectifs de la lecture (correction, compréhension, recherche d'information). Cette expérience a clairement montré que (1) les variables structurelles (structure morphologique et syntaxique) influencent la façon dont les mots sont progressivement transformés en unités de sens et (2) les variables contextuelles comme l'objectif du lecteur (lit-il pour comprendre, corriger, rechercher une information), et le niveau de compréhension recherché, sont également des facteurs qui modifient les stratégies d'analyse perceptive (Pavard, 1983). Par exemple, lors d'une lecture avec l'objectif de comprendre le texte, on observe que plus le lecteur prend en compte le sens global du texte, plus il aura tendance à réduire le processus d'analyse perceptive. Concrètement, on constate qu'il anticipe d'autant plus la fin des mots qu'il comprend le sens du texte. Cet effet était observé sur des mots monomorphémiques, et disparaissait avec des mots polymorphémiques.

Sans entrer dans le détail de l'interprétation, on peut simplement souligner que les processus perceptifs sont déterminés à la fois par l'objectif de la tâche, la structure de la 
représentation externe (ici la structure morphémique des mots), et le contexte plus global dans lequel s'effectue l'activité cognitive (ici le sens donné au texte). D'autres expériences ont également montré l'importance d'autres facteurs contextuels comme la probabilité d'occurrence du mot, sa position dans la phrase, etc. (voir Ségui, 1986 pour une revue sur cette question).

\section{Le contexte cognitif}

Si certaines décisions d'action peuvent se baser uniquement sur une information perceptive, il est clair que beaucoup d'autres nécessitent des traitements de l'information plus élaborés. Que 1'on pense en particulier à toute situation nouvelle ou toute situation anormale rencontrée par un sujet : celui-ci ne peut alors agir sans donner préalablement un sens à cette situation.

On considère en psychologie cognitive que la compréhension d'une situation repose sur des processus interprétatifs, et qu'un sujet pense avoir compris une information, ou plus généralement une situation, quand il a réussi à la rattacher à des informations anciennes déjà connues et acceptées (Haviland \& Clark, 1974, Schank \& Abelson, 1977). Le sujet doit contextualiser toute information nouvelle par des informations anciennes. À un niveau cognitif, les informations qui sont utilisées pour contextualiser et interpréter une information nouvelle proviennent de deux ressources internes :

la mémoire à long-terme : on désigne ainsi l'ensemble des connaissances et des souvenirs que le sujet a conservé. L'une des caractéristiques de cette mémoire est qu'elle est théoriquement illimitée. Une autre de ses caractéristiques est que l'accès aux informations qu'elle contient n'est pas direct : des indices de récupération, qui doivent être présents en mémoire de travail, sont nécessaires. Ces indices de récupération sont des informations associées dans la mémoire à long-terme du sujet à celles qu'il veut récupérer. La base de cette récupération des données mémorisées peut être l'organisation de la mémoire (réseaux sémantiques, schémas, scripts) ou les nombreuses associations entre différents faits créées par l'expérience propre à chacun (c'est ainsi qu'une odeur réactivera un ensemble de souvenirs par exemple). Ces caractéristiques ont des implications sur l'étude du contexte dans les situations de travail. En particulier, il est nécessaire de partir du principe que puisque la récupération des données en mémoire dépend d'associations avec des éléments qui doivent être présents en mémoire de travail, et que ces associations sont au moins pour partie dépendantes de l'expérience de chacun, le contexte cognitif dans lequel un opérateur comprend une situation et prend une décision a toutes les chances d'être au moins partiellement différent de celui de ses partenaires de travail. Il est ainsi possible d'expliquer le fait que face à des situations nouvelles, des pilotes abandonnent leur langage opératif pour utiliser un langage plus riche, caractérisé notamment par des phrases non stéréotypées et des argumentations (Karsenty \& Falzon, 1993).

la mémoire de travail : cette mémoire est limitée, éphémère, et comprend pour cela uniquement les informations issues des derniers traitements effectués. L'accès aux informations qu'elle contient est direct. Les données contenues dans la mémoire de travail proviennent soit de la perception, soit de la mémoire à long-terme. On considère généralement qu'elles sont très dépendantes de l'activité de l'opérateur (Bisseret, 1970). Sperber \& Wilson (1986) associent la mémoire de travail au "contexte immédiat", c'està-dire celui dans lequel les informations entrantes vont être immédiatement comprises. Dans le cadre de leur théorie de la pertinence, cela signifie que l'opérateur n'envisage pas d'autres contextes s'il aboutit à une interprétation pertinente de l'information 
entrante dans ce contexte immédiat. Dans le cas contraire, il dispose de plusieurs mécanismes pour modifier le contexte immédiat. En particulier, ce contexte peut être modifié par récupération de croyances immédiatement associées à celles présentes en mémoire de travail, et stockées en mémoire à long-terme. Le caractère limité et éphémère de la mémoire de travail doit conduire à distinguer ce que le sujet peut voir et entendre, ou vient de voir et vient d'entendre, de son contexte immédiat réel. Par exemple, on ne peut affirmer qu'un opérateur est dans un contexte d'informations données uniquement à partir d'une analyse du contenu de l'écran sur lequel il travaille. En outre, le caractère limité et éphémère de la mémoire de travail peut expliquer pourquoi deux opérateurs disposant des mêmes informations à un moment donné ne comprennent pas de la même façon une nouvelle information : si l'un d'eux a réalisé entre temps une tâche qui a mobilisé sa mémoire de travail, son contenu précédent peut être perdu, et sa compréhension de l'information nouvelle ne se fera pas nécessairement dans le même contexte "immédiat" que son collègue. On peut ainsi rendre compte de certains problèmes d'incompréhension ou d'ambiguïté observés dans des dialogues de travail (ex., Cahour \& Karsenty, 1996).

Nous distinguons dans la suite quatre types d'information qui peuvent résider dans la mémoire de travail, et avoir une influence déterminante sur le travail collectif.

\section{Le contexte des buts}

Le sens donné à une information dépend très largement de la finalité de l'activité. Ceci est dû au fait qu'un but particulier implique des inférences réalisées à partir d'une information donnée. Un agent de maintenance qui constate une panne sur un dispositif technique comprendra par exemple que l'intervention qu'il avait fait sur ce même dispositif technique n'a pas été assez efficace. Un agent de production en conclura, quant à lui, que ses critères de production risquent de ne pas être satisfaits et qu'une intervention de l'équipe de maintenance est urgente. La même information peut donc prendre un sens très différent suivant les acteurs. Dans certains cas, cela peut conduire à des conflits. En reprenant notre exemple, on peut s'attendre à des situations où l'agent de production demande à l'agent de maintenance d'accomplir une action qui rétablisse rapidement la production, alors que l'agent de maintenance préférera rechercher la cause profonde du problème, et n'envisager une action réparatrice qu'ensuite (voir Sauvagnac \& Falzon, 1996).

Un collectif sera d'autant plus efficace que les opérateurs le composant connaîtront les buts particuliers de leurs collègues. Cette efficacité peut notamment se traduire dans les présentations (sous forme orale ou écrite : rapports, comptes-rendus, etc.) faites par les uns aux autres. Une analyse de dialogues de conception a ainsi montré que des concepteurs prenaient en compte les buts de leurs collègues lorsqu'ils décrivaient une solution (Karsenty, 1995) : les parties de la solution qui étaient décrites, ainsi que le niveau de précision auquel elles étaient décrites, s'avéraient directement liés aux tâches de l'interlocuteur.

\section{$\underline{\text { Le contexte des interprétations antérieures }}$}

La mémoire de travail conserve, dans une certaine mesure, les interprétations antérieures. L'utilisation de ces interprétations antérieures est évidente dans le cas de la compréhension des références anaphoriques, pour lesquelles il est toujours nécessaire de se reporter à une autre partie du dialogue pour leur donner une interprétation (extrait 1). On a aussi invoqué son rôle dans l'identification des contenus implicites d'un acte de parole (ex., Sadek, 1996). Par exemple, à un guichet de gare, si un client demande une première fois "Quel est le tarif pour Lyon à $10 \mathrm{~h}$. en première classe ?", et qu'après avoir obtenu une 
réponse, il demande "Et si je pars en seconde classe, c'est combien ?", le guichetier inférera normalement que le client veut toujours partir à Lyon dans le train de $10 \mathrm{~h}$.

\section{Extrait 1 : Exemple de référence anaphorique}

A : Jean a pris Louis et Rémi à la sortie du bureau.

$\mathrm{B}:$ Il voulait les emmener où ?

Dans une étude sur des dialogues d'assistance à l'utilisation d'un logiciel de traitement de texte, Salembier (1993) avait mis en évidence qu'une grande partie des interprétations d'actions du novice faites par l'expert était contextualisée par "l'historique des interactions précédentes". Cette mise en évidence a été possible en demandant à l'expert de commenter après-coup et sur la base d'une réécoute (auto-confrontation) l'interaction qu'il avait eu avec le novice. L'extrait 2 ci-dessous reflète le type de commentaire obtenu, montrant le rôle des interprétations antérieures construites par l'expert.

\section{Extrait 2 (tiré de Salembier, 1993, p.109; souligné par nous) :}

Épisode 1 (l'expert commente l'activité du novice) :

"Là il (le novice) essaie de caler le texte en utilisant le déplacement, mais ça ne marche pas parce qu'il n'y a rien pour maintenir le texte... alors il fait des espaces...il ne connaît sûrement pas la notion de gestion de texte à l'écran"

Épisode 2:

"Alors là, c'est pareil que tout à l'heure sauf qu'il y a une tabulation de posée mais qu'il ne peut pas la voir parce qu'il n'a pas visualisé les caractères cachés ; alors il déplace le texte qui reste aligné parce qu'il y a cette tabulation et on pourrait supposer qu'il connaît la notion. Mais en fait, il ne connait pas ce type de manipulation, à mon avis il ne comprend pas comment ça marche, on l'a vu tout à l'heure...et là c'est juste un coup de chance parce qu'il y avait déjà une tabulation."

Cette même étude a révélé que le rôle exercé par les interprétations antérieures sur l'interprétation d'une information nouvelle est plus de l'ordre de la contrainte que de l'acte totalement délibéré. Ceci peut s'expliquer par un biais inhérent au fonctionnement cognitif, le biais de confirmation : le sujet préférera toujours des interprétations confirmant ses interprétations antérieures (Evans, 1989). Cela le conduit souvent à préférer une explication locale pour rendre compte d'une nouvelle information contredisant ses interprétations antérieures, voire carrément à l'ignorer, plutôt qu'à une remise en cause de ces dernières. L'extrait 3, toujours tiré de l'étude de Salembier, donne un exemple d'explication locale construite par l'expert pour lui éviter de remettre en cause une interprétation antérieure.

\section{Extrait 3 (tiré de Salembier, 1993, p.163) :}

L'expert s'est représenté l'utilisateur comme un expert du logiciel de traitement de texte. Or celui-ci produit un comportement plus caractéristique d'un novice. L'expert commente :

"Là c'est bizarre...parce que ça c'est généralement les personnes qui ne connaissent pas le traitement de texte qui font ça, effacer du texte parce qu'ils sont en mode Surimpression...mais il faut dire que c'est pas évident même quand on connaît Startext de savoir immédiatement si on est en Insertion ou en Surimpression. Des fois, on ne s'en rend pas compte, c'est facile de mélanger, et puis là c'est quelqu'un qui connaît Startext hein..."

\section{$\underline{\text { Le contexte des attentes }}$}

Nous avons vu que des contenus de la mémoire de travail pouvaient provenir de la mémoire à long-terme. Il est ainsi possible d'hériter de l'expérience passée. Cette expérience passée est souvent porteuse d'attentes dans la situation présente. Par exemple, en entrant dans un restaurant, on s'attend à être accueilli et invité à s'attabler ; on s'attend aussi à recevoir un menu, et à pouvoir y faire un choix, etc. Dans le cadre de la conversation, quand on pose une 
question, on s'attend à recevoir une réponse, et quand on salue son interlocuteur pour lui dire au revoir, on s'attend à ce qu'il nous salue en retour.

Beaucoup d'auteurs ont montré le rôle fondamental que jouent les attentes dans le cadre d'une activité coopérative. Les ethnométhodologues, par exemple, ont introduit les notions de "paires adjacentes" (le fait qu'un énoncé en appelle automatiquement ou conventionnellement un autre, comme dans le cas de la question et de la réponse) et de "projectabilité" d'un énoncé (le fait que tout message ouvre un champ de réponses possibles qui va être exploité par les interlocuteurs pour produire et comprendre la réponse effective) pour traiter de la coordination dans la conversation (ex., Sacks, Schegloff \& Jefferson, 1974). De notre point de vue, ces notions renvoient à celle d'attente partagée dans un dialogue. Grice (1975) a proposé une théorie de la conversation fondée sur des maximes conversationnelles qui peuvent être conçues comme les attentes que tout interlocuteur fait normalement en interagissant avec quelqu'un (voir la section dialogue et communication).

Suchman (1987), dans son analyse de l'interaction entre des utilisateurs et un photocopieur "intelligent", a aussi souligné le rôle des attentes des utilisateurs ${ }^{2}$. Elle a montré en particulier l'obstacle que représentent leurs attentes erronées concernant les comportements de la machine. Ainsi, jamais les utilisateurs qu'elle a pu observer n'ont interprété la répétition d'une instruction par le photocopieur comme le signe d'une action erronée de leur part : ceci peut s'expliquer par le fait que les utilisateurs "importent" des attentes issues de l'interaction humaine dans laquelle une telle utilisation de la répétition ne se produit pas. Autre exemple : les utilisateurs anticipent le résultat de leurs actions, et comparent leurs effets réels avec ces attentes pour juger de l'adéquation de ces actions. Quand leurs attentes sont en décalage avec ce que le photocopieur fait réellement, les utilisateurs peuvent ne pas suivre l'instruction suivante et s'arrêter d'agir, et ce même si du point de vue du photocopieur, la séquence d'actions réalisée jusqu'ici est normale (cas que Suchman nomme "fausse alarme").

Dans une activité collective, les attentes erronées sont souvent le signe d'une mauvaise représentation de la compétence et/ou du plan d'action de l'autre ou des autres. Leur identification au cours d'une analyse d'activité peut donc aider à résoudre des problèmes de compréhension et de coordination.

\section{$\underline{\text { Le contexte des alternatives }}$}

Plus un opérateur va acquérir d'expérience dans un domaine donné, plus il sera susceptible de former très vite, c'est-à-dire à partir d'un ensemble restreint d'informations, des attentes sur la globalité d'une situation. Or une nouvelle réalité est rarement totalement identique à une situation déjà rencontrée. L'opérateur expérimenté est par conséquent souvent confronté à une situation tout en en envisageant d'autres possibles.

Être dans un contexte d'alternatives revient à dire que l'état interne de l'opérateur est incohérent : son expérience le conduirait à s'attendre à telle et telle propriété de la situation, et ces propriétés soit n'apparaissent pas soit sont différentes. La situation n'est donc pas totalement compréhensible, et l'opérateur peut alors rechercher une explication des différences perçues (Schank, 1986). Ceci peut poser un problème dans le cadre d'activités

\footnotetext{
${ }^{2}$ Le fait de citer Suchman (1987) dans une section consacrée au contexte cognitif peut paraître surprenant si l'on a compris que le travail de cet auteur visait à nier le rôle des représentations mentales dans l'activité. Une telle interprétation du travail de Suchman est cependant erronée (Suchman, communication personnelle), ce que traduit non seulement ses analyses de dysfonctionnements de la communication homme-machine (voir l'exemple que nous rapportons), mais aussi la conclusion de son travail. L'auteur propose en effet une nouvelle théorie de l'action, fondée sur des interactions locales avec l'environnement, lesquelles sont orientées par des représentations abstraites de la situation et d'actions qui aident à exploiter les contingences de l'environnement (cf. p. 188, souligné par nous).
} 
collectives, lorsque l'opérateur ne trouve pas d'explication, ou ne trouve pas l'explication exacte.

Une étude sur la réutilisation de solutions passées dans un bureau d'étude en conception mécanique illustre ces difficultés (Karsenty, 1996a). On a constaté que les concepteurs ayant à réutiliser une solution passée, élaborée par d'autres, se posaient très souvent une question du type : "Pourquoi ont-il fait ainsi et pas autrement ?" L'absence de réponse à cette question produit des effets différents suivant que le concepteur qui se la pose a une très grande expérience dans le bureau d'étude (entre cinq et vingt-cinq ans) ou non. Les concepteurs très expérimentés considèrent généralement l'autre solution possible comme meilleure que celle qui leur est présentée. Par conséquent, l'absence de réponse les conduit généralement à délaisser la solution passée et à préférer celle qu'ils ont spontanément évoqué. Dans certains cas, ce choix peut être erroné. Les concepteurs avec qui nous étions en contact ont rapporté que c'est souvent au bout de plusieurs semaines, voire de plusieurs mois de travail sur la solution préférée qu'ils pouvaient s'apercevoir qu'elle présentait un inconvénient majeur. L'impasse dans laquelle il se trouvait pouvait, dans certaines circonstances, leur faire comprendre la raison exacte de la solution passée qu'ils avaient précédemment délaissée, et éventuellement les conduire à modifier leur décision initiale de rejet de la solution passée. À l'inverse, les concepteurs moins expérimentés vont le plus souvent préférer reprendre la solution passée, peut-être par manque de confiance en eux. Mais là aussi, cette stratégie peut produire des erreurs, quand la solution passée est réellement inadaptée pour satisfaire les nouvelles exigences du projet.

Une autre étude sur l'utilisation de check-lists dans l'aéronautique a conduit aux mêmes conclusions (Karsenty \& al., 1995) : des distorsions dans l'utilisation prévue des check-lists liés aux pannes peuvent être dues au fait que les pilotes se remémorent d'autres procédures déjà appliquées (même sur d'autres avions), et ayant produit le résultat attendu. Lorsque les pilotes ne comprennent pas les raisons des actions qui leur sont prescrites, ils préfèrent dans un certain nombre de cas appliquer la procédure remémorée, ou une combinaison entre cette procédure remémorée et la procédure prescrite.

\section{Dialogue et communication}

\section{$\underline{\text { La multimodalité des actes de communication }}$}

Dans les dialogues de travail, la communication est parfois uniquement verbale. C'est le cas des dialogues d'assistance téléphonique par exemple. Mais lorsque le face-à-face est possible ou que le travail collectif se situe dans un même lieu, elle est toujours multi-modale, alliant la parole aux gestes, regards et postures du corps (ex., Heath \& Luff, 1994, Heath, Luff $\&$ Nicholls, 1995), ou encore à certains artefacts et autres ressources environnementales (ex., Hughes, Randall \& Shapiro, 1992). Généralement, il existe un rapport de contextualité entre les modalités employées pour communiquer : l'une d'elle véhicule une information qui ne prend sens que dans le contexte des autres. Ainsi, l'assertion "il n'est pas totalement circulaire" ne pourra être comprise que si l'interlocuteur a accès au geste fait par le locuteur et désignant du doigt un cercle sur un tableau.

Dans une étude sur la coordination entre contrôleurs aériens (Bressolle, Pavard, \& Leroux, 1995), nous avons analysé le rôle d'un ensemble d'objets et d'outils de représentation de l'information entourant les agents coopérant. La situation étudiée concerne la coopération entre deux agents, le contrôleur organique et le contrôleur radar, chargés de réguler le contrôle aérien. Ces deux agents disposent à la fois des ressources de la communication verbale, du 
fait de leur relation de proximité, mais également des ressources caractérisées par l'ensemble des objets participant à leur environnement : écran radar, "strips" papiers (sorte de bandelettes de papier présentées sur des supports physiques sur lesquelles sont inscrites des informations propres à chaque avion), etc. Une analyse détaillée des actes de communication a montré que la connaissance commune indispensable à une bonne coopération passe par une interprétation par les agents du sens véhiculé par ces objets. Ainsi, les "strips" papier sont positionnés de telle sorte sur la table de travail qu'ils sont utilisés par les agents aussi bien comme support à la mémorisation qu'à la résolution de problèmes ou à la coopération (voir figure 3 ).

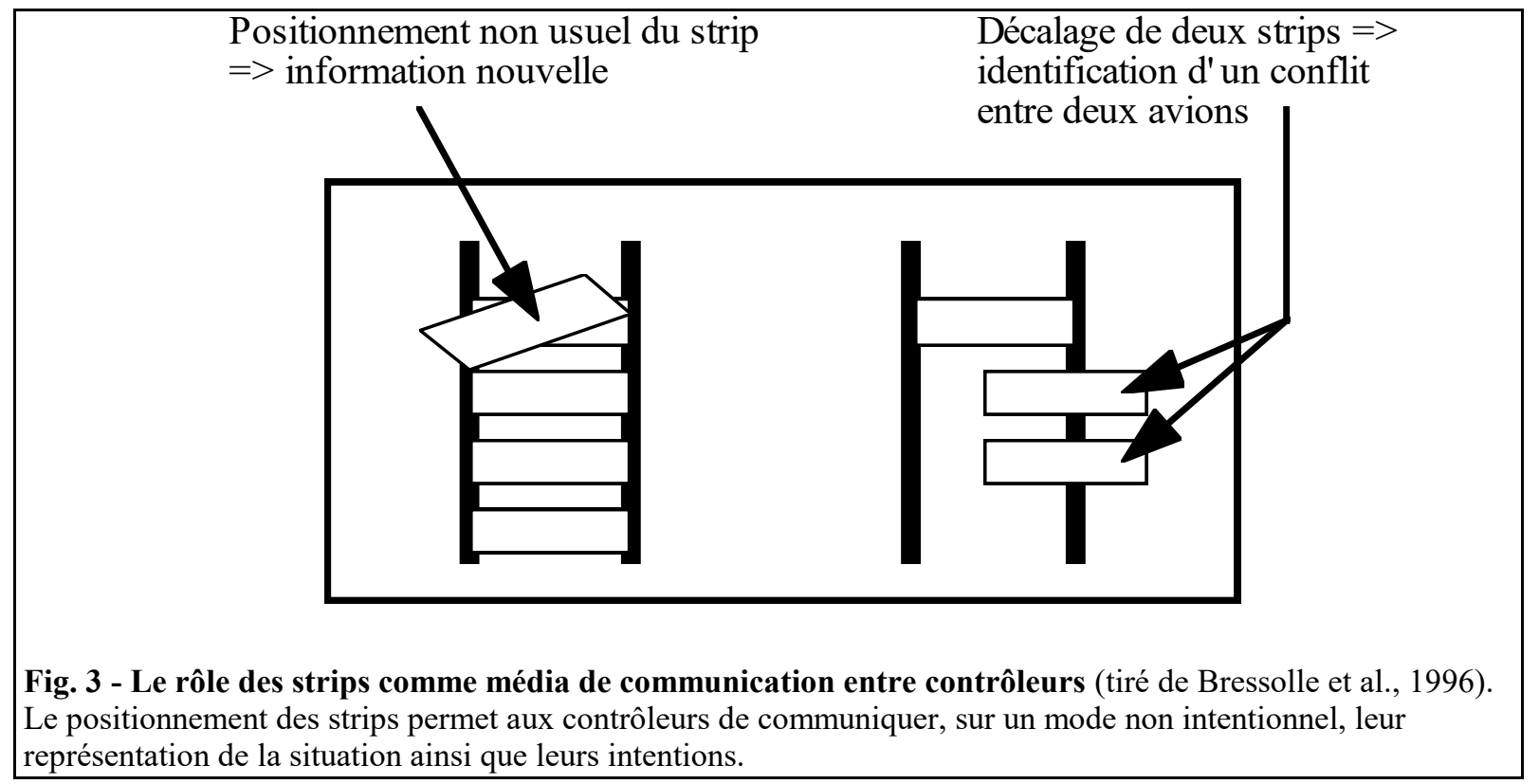

Paradoxalement, ces fonctions des strips n'apparaissent pas explicitement comme telles dans la définition des tâches des contrôleurs. Seule une analyse détaillée des activités des contrôleurs par rapport à ces objets a pu révéler leur importance pour la réalisation de la tâche.

\section{$\underline{\text { La représentation de l'autre }}$}

Des recherches en intelligence artificielle sur les "modèles d'utilisateurs" ont introduit et montré l'intérêt de cette forme de contexte pour la gestion des dialogues (Kobsa \& Wahlster 1989, Cahour \& Paris 1991). Ces recherches ont été confirmées par des études empiriques de dialogues humains, qui ont notamment montré l'influence de la représentation de l'autre sur la prise d'initiative dans le dialogue (Cahour, 1991), et sur la régulation de l'aide apportée à des nouveaux utilisateurs d'un logiciel (Salembier, 1993). Le rôle d'une représentation de l'autre sur le type de langage et la complexité des phrases utilisées a aussi été observé dans le cadre du dialogue homme-machine, l'autre étant dans ce cas la machine (ex., Amalberti, Carbonell \& Falzon, 1993). La plupart de ces travaux ont souligné une dimension en particulier de la représentation de l'autre : la perception de son niveau d'expertise. Par exemple, plus l'interlocuteur est perçu comme expert, plus le locuteur lui laissera l'initiative de contrôler le cours du dialogue (Cahour, 1991).

La situation sociale des interlocuteurs (statuts, rôles, relations hiérarchiques entre les interlocuteurs...) influencent également la production et la compréhension des énoncés. Dans l'extrait 5 , où deux collègues $\mathrm{A}$ et $\mathrm{B}$ dialoguent, $\mathrm{A}$ détient une position hiérarchique nettement supérieure à B. La plupart des interventions de B peuvent alors être interprétées par A comme 
des requêtes de confirmation, alors qu'elles apparaitraient comme des assertions/propositions de solution dans le cas contraire.

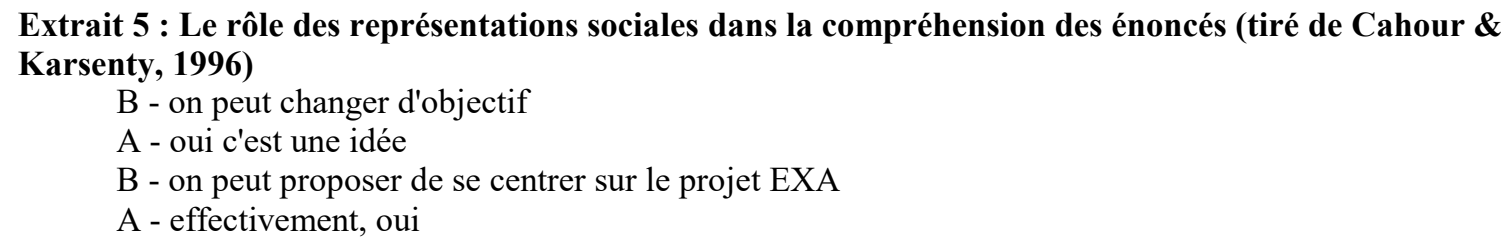

Il faut noter que l'on peut construire non seulement une représentation des caractéristiques de l'autre, mais aussi une représentation de l'image que l'autre a de soi. Clark \& Marschall (1981) montrent comment deux personnes parlant d'un film dont ils ont lu chacun de leur côté l'annonce dans le journal utilisent ce type de croyances complexes (comme par exemple "je sais qu'elle sait que j'ai vu l'annonce") pour choisir leurs expressions référentielles. Par exemple, tandis que l'annonce indique que le film prévu est remplacé par un autre, si $\mathrm{A}$ demande à $\mathrm{B}$ "as-tu déjà vu le film qui passe au cinéma ?", à quel film B va-t-il penser que A fait référence ? Cela dépendra du modèle de son interlocuteur, mais aussi du modèle qu'il pense que son interlocuteur a de lui.

\section{$\underline{\text { Les normes communicationnelles }}$}

Si quelqu'un dit "Mon oncle n'arrive que demain", c'est généralement qu'il pense que l'autre connaît l'oncle en question. Si A s'adresse à B en lui disant "Je n'ai plus d'essence" et que B répond "Il y a un garage au coin de la rue", A comprendra normalement qu'il y a un garage au coin de la rue, mais aussi que ce garage est ouvert et vend de l'essence (Grice, 1975, p.51). Ces exemples de comportement langagier montre que l'intelligibilité mutuelle dans un dialogue repose sur des contenus implicites. Dans le premier exemple, c'est un présupposé qui est implicite. Dans le second, c'est un ensemble d'implicitations liées à la situation de dialogue.

Pour rendre compte de cette part d'implicite, et expliquer comment des interlocuteurs se comprennent dans ces conditions, Grice (1975) a amené l'idée que toute conversation s'établit dans un cadre conversationnel qu'il définit sous la forme d'un Principe Coopératif. Il énonce ce principe de la façon suivante : "chaque partenaire doit contribuer conversationnellement de manière à correspondre aux attentes des autres interlocuteurs en fonction du stade de la conversation, du but et de la direction de l'échange." Grice décompose ce principe en plusieurs maximes de coopération qui précisent comment des interlocuteurs peuvent le respecter. Ces maximes sont :

Maxime de quantité : l'intervention doit apporter suffisamment d'informations ; elle ne doit pas apporter plus d'informations que nécessaire.

Maxime de qualité : ne rien dire que l'on croit faux ; ne rien dire que l'on puisse démontrer.

Maxime de pertinence : l'information donnée doit être pertinente.

- $\quad$ Maxime de manière : éviter d'utiliser des expressions obscures ou ambiguës ; être bref ; donner les informations dans le bon ordre.

Ces maximes jouent le rôle d'attentes socialement partagées : l'interlocuteur s'attend, dans des conditions normales de dialogue, à ce qu'un locuteur soit informatif, pertinent, clair, etc., et le locuteur le sait. Ces maximes sont normatives (et non prescriptives), ce qui signifie que le locuteur peut ne pas les respecter. Mais ce sera alors dans le but de transmettre implicitement un certain message qui correspond à l'explication de pourquoi le locuteur n'a 
pas respecté une certaine maxime. Ainsi, si A pose la question "Auriez-vous l'heure s'il vous plaît ?", et reçoit de B la réponse "non" alors que manifestement B a une montre et sait que A le sait (violation de la maxime de qualité), A en inférera probablement que B ne veut pas lui donner l'heure, et que c'est ce qu'il a voulu lui signifier par sa réponse.

La prise en compte de ce contexte quasi institutionnel que représentent le principe coopératif et les maximes conversationnelles s'est avérée importante dans les projets visant à concevoir des dialogues coopératifs homme-machine, que ce soit pour essayer de faire respecter les maximes par la machine (ex., Sadek, 1996), ou pour étudier dans quelle mesure ces maximes sont directement applicables ou non dans le cadre du dialogue homme-machine (Bernsen, Dybkjaer \& Dybkjaer, 1996).

\section{La culture}

On a déjà beaucoup insisté sur le fait que l'appréhension d'une situation repose sur des processus interprétatifs. Les niveaux de contexte considérés jusqu'ici conduisent à préciser que l'interprétation exploite des représentations externes ou internes. En réalité, le sens donné à une situation repose aussi, et de façon primordiale, sur un ensemble de croyances implicites formant la culture de chacun. Ces croyances implicites se distinguent des représentations abordées précédemment dans la mesure où elles sont partagées et mutuellement admises par les membres d'un collectif. Pour cette raison, elles ne sont généralement pas soumises au jugement, et sont acceptées comme des faits non contestables.

Prenons un exemple où la culture intervient dans la formation de la représentation d'un problème (tiré de Winograd \& Florès, 1987) : Madame X a l'habitude d'aller à son travail en voiture. Un jour, sa voiture tombe en panne. Elle apprend que la réparation va être très coûteuse. Elle envisage alors trois alternatives, qu'elle considère comme les seules possibles : soit elle accepte la réparation, soit elle achète une nouvelle voiture, soit elle se rend désormais à son lieu de travail en transport en commun. Si Madame X n'envisage pas d'autres alternatives, c'est parce qu'elle est influencée par une croyance implicite propre à sa culture : pour travailler, on doit se rendre sur son lieu de travail. Cette croyance sous-tend la formulation des trois alternatives, mais empêche aussi d'en envisager d'autres (travail à domicile par exemple). Son intervention dans la formation de la représentation de son problème n'est pas délibérée, contrairement à son jugement concernant les trois alternatives.

L'étude de la culture s'avère fondamentale dans les situations de transferts technologiques. Une démarche anthropotechnologique est née de cette préoccupation, prenant sa source dans l'analyse ergonomique du travail développée en particulier au sein du laboratoire d'ergonomie du CNAM (Wisner, 1997). L'anthropotechnologie a pour but de transférer des technologies ou des systèmes organisationnels dans des pays de cultures différentes. Dans cette perspective, la dimension culturelle joue le rôle de contexte dans lequel doit être repensé le dispositif technique. Cette démarche peut d'ailleurs être appliquée dans le cadre de la conception à l'intérieur d'un même pays ou d'une même entreprise, sachant que la notion de culture locale peut également jouer un rôle important dans le mécanisme de rejet ou d'acceptation de tout dispositif technique ou communicationnel.

Afin d'illustrer cette dimension anthropologique, nous pouvons citer une étude récemment faite pour le transfert de la technologie des marais salants de l'Ouest de la France vers les populations Susu de Guinée (Geslin, 1997). Pour les Susu, le sel est avant tout le produit de la filtration de l'eau de mer à travers la terre salée. L'innovation proposée ne prenait pas en compte ce type de représentation propre à la culture locale. Elle proposait la mise en œuvre de marais salants en prise directe sur la mer. Les populations Susu ont très rapidement conclu que le sel ainsi obtenu ne pouvait pas être du sel puisqu'il manquait un "catalyseur" 
dans sa conception, la terre. L'étude a révélé que ce facteur culturel avait contribué au rejet partiel de l'innovation proposée.

De nombreux projets liés à l'introduction de technologies modernes peuvent se heurter à ce type de difficulté : téléphones portables difficiles à être acceptés dans certains milieux sociaux, fonctionnalité des répondeurs téléphoniques devant être compatibles avec les normes de politesse des pays dans lesquels ils sont vendus, systèmes organisationnels et relations hiérarchiques propres à chaque pays, etc. La mise en place de nouvelles technologies de l'information et de management peut également se heurter à de nombreux problèmes culturels : comment passer d'un management basé sur la compétition de groupes à un management basé sur une culture de coopération et de partage de connaissances ${ }^{3}$.

\section{La structure organisationnelle}

La conception d'un point de vue organisationnel de systèmes coopératifs se heurte à une difficulté majeure : elle doit faire appel à des procédures explicites (allocation de rôles, de responsabilités, procédures de coordination, etc.) alors que simultanément, nous savons que le propre de toute activité coopérative est de laisser, dans le cadre des procédures explicites, une libre place à des activités implicites seules capables de permettre au groupe de répondre de façon originale et créative aux situations nouvelles.

De façon générale, on peut considérer que tout système socio-technique complexe est associé à deux modes de fonctionnement complémentaires : un mode explicite, qui peut être défini par des règles ou procédures de fonctionnement, et un mode implicite, qui est synergique, et qui recouvre en grande partie le contexte dans lequel se déroule l'action.

L'analyse détaillée des modes de fonctionnement de tels systèmes complexes révèle à quel point la partie implicite peut jouer un rôle fondamental dans la compréhension systémique de la coopération et dans le processus de conception d'outils informatiques d'aide à la coopération.

Par exemple, dans une étude faite sur la coopération dans une petite équipe devant répondre à des situations urgentes (SAMU de Corbeil), nous avons montré que les principaux mécanismes de coopération étaient soit liés à des processus de communication pluri-adressée (un locuteur s'adressant simultanément à plusieurs auditeurs mais de façon non intentionnelle) soit liés à des modes de fonctionnement opposés aux règles explicites et ceci dans l'intérêt de la communauté et de l'efficacité du groupe (Pavard, Benchekroun, \& Salembier, 1990). Par exemple, les permanencières qui reçoivent les appels pouvaient prendre des décisions médicales, alors que cela est strictement interdit, la décision médicale devant être prise par les médecins. Dans ces deux cas de figure, une structure organisationnelle implicite s'était mise en place sans que les acteurs eux-mêmes soient conscients de son importance d'un point de vue fonctionnel. Il est donc évident que dans ce type de situation, il serait très risqué d'entreprendre une spécification fonctionnelle uniquement sur la base d'une description des procédures explicites ou d'une représentation qui ne s'appuierait pas sur une analyse approfondie des activités des agents. Le taux d'échec très élevé de systèmes informatiques d'aide au travail coopératif (collecticiels) est dans la majorité des cas lié à une sous-estimation du rôle de cette activité implicite.

Dans un autre domaine, qui est celui du contrôle aérien, nous avons pu mettre en évidence le même type de phénomène. L'activité de contrôle aérien est d'une certaine façon

\footnotetext{
3 Voir Giga Information Group : information@gigaweb.com, pour un exemple de changement radical de fonctionnement d'une entreprise du fait de l'introduction d'une culture d'entreprise basée sur la coopération individuellement désintéressée.
} 
extrêmement formalisée, notamment pour tout ce qui concerne les communications entre sol et bord. Le vocabulaire et la phraséologie pour transmettre des instructions sont totalement définis. Par exemple, pour transmettre une instruction de changement de cap, le contrôleur doit employer impérativement une expression respectant le format suivant : "AF 018 turn left heading 215" (ce qui se traduit par : Avion Air France 018, tournez à gauche cap 215). Pour réaliser son activité, le contrôleur est assisté par un autre agent (le contrôleur organique), qui l'aide à mieux anticiper le trafic et à le gérer. L'efficacité du contrôleur principal dépend donc étroitement des processus de coopération, lesquels ne sont absolument pas formalisés. Dans de telles conditions, il est possible de mettre en évidence que la phraséologie peut subir des altérations profondes en fonction du niveau de charge de travail : des règles implicites prennent alors la place des règles explicites, et ce dans le but de s'adapter à une "demande" particulière de la situation. Une analyse détaillée des modes de communication montre également que, dans de tels systèmes, les acteurs mettent eux-mêmes en place des processus de communication qui ont pour objectif d'enrichir le corps de connaissances communes, permettant ainsi au système collectif de mieux "répondre" à des situations imprévues. Nous avons pu mettre en évidence, à partir d'une analyse des communications en période d'incidents, que la récupération de situations incidentelles était possible grâce aux informations délibérément "injectées" dans le système technique alors que les procédures officielles ne l'imposent pas. Un des processus le plus efficace est le pluri-adressage qui permet à plusieurs agents d'écouter une conversation initialement destinée à une seule personne. La fiabilité globale du système technique est donc à la fois liée aux procédures explicites et aux processus informels rendant l'information critique redondante (Bressolle, Decortis, Pavard \& Salembier, 1996).

Nous retiendrons de ces deux études que tout système organisationnel peut être vu comme la superposition de deux modes de fonctionnement explicites et implicites pouvant être synergiques. Les mécanismes de fonctionnement implicite sont souvent liés à la prise en compte d'informations contextuelles dont le flux n'est pas et ne peut pas être contrôlé par des règles de fonctionnement formalisables.

\section{Le contexte supposé partagé}

Dans l'étude des activités collectives, la problématique du contexte est intimement liée à celle du contexte partagé. La raison en est simple : l'intelligibilité mutuelle des actions de chacun repose sur le partage du contexte. Par exemple, A ne peut pas comprendre ce que B signifie en disant "J'essaye d'imprimer, mais ça ne fonctionne pas", s'il n'a aucun moyen de savoir que A est installé devant son ordinateur avec lequel il vient de rédiger un texte, et veut l'imprimer. De même, un pilote ne comprendra pas que le copilote modifie le niveau de vol, alors que ce n'était pas initialement prévu, s'il ne sait pas que le centre de contrôle vient de lui demander de modifier son plan de vol pour laisser passer un autre avion.

La notion de contexte partagé a été envisagée de différents points de vue : connaissances mutuelles chez les philosophes du langage (ex., Smith, 1982, Grice, 1975) ou encore chez certains psychologues de la communication (Clark \& Marshall, 1981 ; Krauss \& Fussell, 1990, Gibbs \& Mueller, 1990) ou environnement cognitif partagé (Sperber \& Wilson, 1986), selon notamment que l'on accepte l'idée d'un corps de connaissances communes stabilisées, ou, à l'inverse, uniquement l'idée d'une intersection nécessaire entre les environnements (contextes) cognitifs individuels. Cette dernière position s'intègre à une théorie rejetant une vision de la communication comme un processus d'encodage-décodage, qui vise à rendre compte d'une interprétation univoque du message transmis, pour affirmer le rôle des processus inférentiels nécessairement imparfaits. 
Tout en nous inscrivant dans ce cadre théorique, l'examen empirique de dialogues nous conduit à dépasser la seule idée d'une intersection entre les contextes individuels, pour invoquer une notion de contexte supposé partagé (voir aussi Flahault, 1982). En effet, c'est précisément ce qui est non partagé et cru à tort partagé par les interlocuteurs qui permet d'expliquer nombre de difficultés d'intelligibilité mutuelle. L'illusion de partager un même contexte est source d'incompréhension et de malentendu (voir extrait 4). De façon générale, cela s'explique par le fait que supposer un contexte partagé conduit le locuteur à laisser implicite un certain nombre de contenus. Le message produit est de ce fait ambigu, c'est-àdire ouvert à plusieurs interprétations possibles. L'incompréhension ou le malentendu se produit dès que l'auditeur interprète un tel message dans un contexte différent de celui qui est envisagé et supposé à tort partagé par le locuteur.

\section{Extrait 4 : Illustration d'un contexte supposé partagé à tort (tiré de Cahour \& Karsenty, 1996)}

Le contexte de l'échange qui suit est le suivant : E est un ancien étudiant qui cherche à obtenir un poste universitaire, et $\mathrm{P}$ un professeur ; ils se sont déjà rencontrés. E téléphone à $\mathrm{P}$ pour lui demander une lettre de recommandation. Une tierce personne, Y, qui est mentionnée par P, connaît bien E puisqu'ils travaillent ensemble.

\footnotetext{
E1 - Bonjour monsieur, je suis monsieur "E";

P1 - bonjour

E2 - Je vous téléphone pour savoir si vous pourriez me faire une lettre de recommandation pour un poste universitaire.

P2 - oh...oui...euh...mais...je ne comprend pas bien... je ne sais pas si nous sommes sur la même longueur d'onde...j'ai eu monsieur $\mathrm{Y}$ au téléphone, il m'a dit que vous souhaiteriez avoir ce poste dans notre laboratoire

E3 - Ah je ne savais pas!... mais depuis, j'ai appris que les laboratoires possibles sont prédéfinis et votre laboratoire n'est pas sur la liste.
}

Commentaire : Comme on peut le constater, P exprime en P2 une incompréhension face à la demande de E. On comprend ensuite que cette incompréhension est causée par la contradiction entre la demande de $\mathrm{E}$ et l'attente qu'avait formé $\mathrm{P}$ concernant la demande de $\mathrm{E}$ : $\mathrm{P}$ s'attendait à ce que $\mathrm{E}$ lui demande un accord concernant sa candidature dans son laboratoire, alors que la demande réelle de E porte uniquement sur une lettre de recommandation. On comprend aussi, même si cela n'est pas totalement explicite, que $\mathrm{P}$ a pu s'attendre à ce qu'E lui demande un accord concernant sa candidature dans son laboratoire parce qu'il sait que $\mathrm{Y}$ et $\mathrm{E}$ travaillent ensemble, et parce qu'il a dû en inférer que $\mathrm{Y}$ et $\mathrm{E}$ avaient parlé ensemble du coup de téléphone de $\mathrm{Y}$ à $\mathrm{P}$. En d'autres termes, le dysfonctionnement est causé par le fait que $\mathrm{P}$ attribue à $\mathrm{E}$ une histoire qu'il n'a pas eue (la rencontre entre Y et E), et utilise cette croyance pour contextualiser la demande de E.

Du point de vue de l'analyse de l'activité collective, il peut être nécessaire d'identifier les situations où le contexte supposé partagé par l'un ne l'est pas en réalité, et tenter de comprendre la source de ce type d'erreur. Voici un exemple d'étude dont l'objet était de comprendre la source de malentendus dans des dialogues d'assistance à distance.

Cet exemple provient d'une étude des processus de référenciation dans le dialogue (Giboin, 1997). La situation étudiée par l'auteur est une situation de dialogue d'assistance à distance, dans laquelle un utilisateur novice d'un logiciel de traitement de texte se fait aider par un expert pour réaliser certaines tâches. L'auteur analyse un dysfonctionnement de la communication qui survient alors que l'expert indique au novice comment fermer une fenêtre : "Alors pour fermer la fenêtre, c'est le petit carré qui est en haut à gauche...tu cliques dessus." Il faudra 19 échanges pour que le novice réussisse à déterminer de quel "petit carré" l'expert voulait parler, et ferme sa fenêtre. L'analyse a permis de révéler que cette difficulté à communiquer était en partie due au fait que l'expert a mis très longtemps avant de comprendre que le novice avait essayé d'identifier le "petit carré en haut à gauche" dans une zone de l'écran différente de celle qui était envisagée par l'expert. Autrement dit, l'expert supposait à tort que son contexte perceptif était partagé avec le novice. 
L'analyse menée par Giboin va plus loin et tente d'expliquer pourquoi le novice n'a pas fixé son attention sur la bonne zone de l'écran. La première raison qu'il identifie est qu'il existe d'autres "petits carrés" sur l'écran. Le novice s'était fixé sur un autre de ces petits carrés, en fait un très petit carré. Pour expliquer pourquoi celui-ci et pas un autre, l'auteur remarque que dans le dialogue antérieur entre l'expert et le novice, d'autres "petits" objets avaient été mentionnés par l'expert. Il note alors que ces "petits" objets ont une taille similaire au "petit" carré incorrectement identifié par le novice, et fait l'hypothèse que cette expérience du "petit" explique au moins en partie l'erreur du novice. En reformulant cette analyse dans le cadre présenté dans cet article, on dirait que le choix d'un contexte perceptif par le novice a été influencé d'une part par la structure de son environnement externe (plusieurs petits carrés présents), d'autre part par sa mémoire du dialogue avec l'expert (autres énoncés référant à de "petits" objets).

\section{Ajustement contextuel et explication}

Jusqu'ici, on a pu donner une vision du contexte interprétatif comme quelque chose qui s'impose aux opérateurs. L'analyse du contexte dans le cadre d'un collectif de travail livre en réalité une autre vision : le contexte interprétatif est l'objet d'ajustement.

Les ajustements contextuels sont observables dans le dialogue, quand une information apportée par l'un n'est pas directement compréhensible par l'autre ou contredit certaines de ses croyances. Typiquement, ce type de situation fait naître un besoin d'explication. L'exemple suivant, tiré d'une conversation réelle, en est une illustration.

\section{Extrait 5}

$\mathrm{B}$ vient d'annoncer à $\mathrm{A}$ au cours d'une conversation que Jean a vendu sa voiture.

A1 Pourquoi Jean a vendu sa voiture?

B1 Je crois que c'est parce qu'il divorce.

A2 Tu veux dire, pour partager l'argent avec sa femme ?

B2 Ouais, la voiture a rapporté 80000 francs.

A3 Mais je croyais que sa femme était riche, je ne vois pas pourquoi elle aurait besoin de ça!

B3 Elle était riche. Mais elle ne l'est plus, justement.

A4 Ah bon ...je ne savais pas.

B4 Voilà.

Dans cet exemple, A a une difficulté à comprendre une nouvelle information ("Jean a vendu sa voiture"). La raison la plus probable pour rendre compte de cette difficulté est que le contenu de sa mémoire de travail, composé notamment des éléments de connaissance associés aux concepts "Jean", "vente", "vente-de-voiture", et "voiture", ne permet pas de rendre cette nouvelle information cohérente avec les informations déjà connues par A sur Jean. En d'autres termes, A ne voit pas, étant donné ce qu'il sait sur Jean, ce qui aurait pu amener ce dernier à vendre sa voiture. L'explication B1 vient modifier ce contexte initial, en corrigeant une croyance antérieure de A ("Jean n'est plus marié, il divorce"). La phase d'ajustement contextuel aurait pu s'arrêter ici, mais on constate que cette nouvelle information apportée par $\mathrm{B}$ s'avère elle aussi difficile à assimiler par $\mathrm{A}$. La suite du dialogue révèle la raison de cette difficulté : cette nouvelle information contredit une croyance de A (voir A3 "Mais je croyais que sa femme était riche, je ne vois pas pourquoi elle aurait besoin de ça !"). L'explication en B3 vise à modifier cette croyance erronée (B3 : "Elle était riche, mais elle ne l'est plus justement"). A, qui partage maintenant avec B un ensemble de représentations (sur Jean, sa femme, etc.), semble alors accepter l'information initiale que celui-ci voulait transmettre ("Jean a vendu sa voiture").

L'enjeu d'une explication est l'ajustement des contextes interprétatifs des participants au dialogue (Karsenty \& Brézillon, 1995, Karsenty, 1996b). Cet ajustement contextuel 
implique généralement des modifications dans le contexte initial de l'acteur ayant émis un besoin d'explication.

Assez fréquemment, ces modifications du contexte de l'interlocuteur ne sont possibles que parce que celui-ci va rendre explicite son propre contexte interprétatif initial, c'est-à-dire ses représentations subjectives associées à la situation de dialogue présente. L'extrait 5 cidessus en donne une illustration, quand A énonce en A3 "Mais je croyais que sa femme était riche, je ne vois pas pourquoi elle aurait besoin de ça !" Cet énoncé rend explicite une partie du contexte de $\mathrm{A}$, constituée d'une interprétation antérieure récupérée de sa mémoire à longterme. B peut alors facilement la corriger.

Les dialogues explicatifs, qui traduisent un processus d'ajustement contextuel, sont une occasion pour des gens travaillant ensemble d'accéder aux représentations mentales que chacun d'eux peut former face à une situation de travail donnée, et qui ne sont pas nécessairement partagées. Dans cette optique, l'explication peut être envisagée comme un outil de coordination du travail collectif.

\section{Conclusion}

Notre réflexion est née du constat que la notion de contexte est trop floue pour être utilisée dans son acception globale. Il est par conséquent nécessaire de la rendre plus opérationnelle en l'analysant à différents niveaux conceptuels. Nous avons proposé dans cet article différents niveaux d'analyse du contexte : environnement externe, niveaux perceptif, cognitif, communicationnel, culturel, organisationnel. Nous avons en outre insisté sur l'existence de contextes supposés partagés, et la mise en œuvre de processus d'ajustement contextuel au sein d'un collectif.

Que révèle cette diversité de la notion de contexte ? Il apparaît, suite aux exemples que nous avons analysé, que le concept de contexte est lié à la notion de complexité. Tout système complexe ne peut être identifié à un système fermé (comme dans l'approche représentationnelle externe développée par Zhang et Norman) dont il serait possible d'identifier de façon exhaustive les variables qui, à un moment donné, sont susceptibles d'expliquer tous les phénomènes pertinents. Une situation de travail, et a fortiori une situation de travail collectif, est un système ouvert, jamais totalement prédictible, et déterminé par un nombre difficilement délimitable de facteurs contextuels.

D'un point de vue méthodologique, ceci nous amène à faire trois propositions :

1. Toute méthodologie s'intéressant à l'analyse des activités collectives doit combiner à une approche déterministe (par exemple l'approche naturaliste comme en sociologie ou en psychologie expérimentale), qui a pour objectif de représenter le système étudié par un nombre prédéfini de variables, une approche de type ethnométhodologique, qui a pour caractéristique d'analyser le cours des activités de façon ouverte, c'est à dire sans limitation a priori sur les facteurs pouvant expliquer l'influence du système ou l'enchaînement des activités. C'est cette approche ethnométhodologique qui apportera des éléments informatifs importants sur la notion de contexte et la façon dont ce contexte peut influencer au cours du temps la prise de décision. En aucun cas, nous ne pensons qu'une approche uniquement expérimentale (telle qu'elle s'est développée par le courant "Human Factors" nord-américain par exemple) peut répondre de façon adéquate aux exigences des situations de travail réelles.

2. De façon générale, le contexte doit être analysé à plusieurs niveaux allant des niveaux perceptifs aux niveaux culturels. Il est en outre nécessaire de prendre en compte le fait que ces différentes couches de contexte peuvent entrer en interaction les unes avec les autres. Bien sûr, suivant les situations, il ne sera pas nécessaire de porter son attention 
sur tous ces niveaux (par exemple, dans une situation de dialogue téléphonique simple, l'analyse de l'environnement externe n'a pas lieu d'être). Mais notre proposition méthodologique est ici de systématiser l'analyse du rôle du contexte à tous les niveaux, en adoptant le moins d'a priori possibles sur les niveaux pertinents ou non pertinents à prendre en compte. Une attention particulière devra également être portée sur la dimension culturelle car elle échappe par définition à une "auto-analyse" (même par un autre sujet appartenant à la même culture). En effet, immergée dans notre propre culture, il nous est impossible d'en identifier les caractéristiques profondes. Ce n'est que par comparaison avec d'autres cultures, ce qui est l'approche de l'anthropotechnologie, que l'on peut identifier la dimension culturelle d'une activité à caractère pratique ou symbolique.

3. Enfin, retenons que l'un des principaux freins à une bonne communication au sein d'un collectif est l'incapacité d'interagir avec autrui. L'interaction fournit une occasion de détecter des écarts dans les contextes individuels, et de les effacer. Elle est donc un moyen d'éviter des problèmes de coordination. Elle est aussi un moyen de construire une culture commune. Cette réflexion prend tout son intérêt à l'heure où l'on veut promouvoir le télétravail et la décentralisation. Certes, ces nouvelles orientations peuvent répondre à certains besoins socio-économiques, mais leur réussite réelle supposera (notamment) que les acteurs placés à distance gardent les moyens d'ajuster facilement leur contexte, c'est à dire leur vision de la tâche, de l'environnement de travail, de l'autre, de l'image que l'autre a de soi, etc. Les premières études sur les téléacteurs semblent montrer qu'il existe actuellement des lacunes de ce point de vue (Performances Humaines et Techniques, 1997).

\section{Bibliographie}

Amalberti R., Carbonell N. \& Falzon P. (1993) User representations of computer systems in human-computer speech interaction. International Journal of Man-Machine Studies, 38, 547-566.

Bernsen N.O., Dybkjaer H. \& Dybkjaer L. (1996) Cooperativity in Human-Machine and Human-Human Spoken Dialogue. Discourse processes, 21, 213-236.

Bisseret A. (1970) Mémoire opérationnelle et structure du travail. Bulletin de Psychologie, 24(5-6), 280-294.

Bressolle, M.C., Pavard, B. \& Leroux, M. (1995) The role of multimodal communication in cooperation and intention recognition : the case of air traffic control. Proceedings of the International Conference on Cooperative Multimodal Communication, Theory and Applications, Eindhoven, The Netherlands, 24-26 May 1995.

Bressolle, M-C., Decortis, F., Pavard, B. \& Salembier, P. (1996) Traitement cognitif et organisationnel des micro-incidents dans le domaine du contrôle aérien : analyse des boucles de régulation formelles et informelles. In : De Terssac G. and Friedberg E. (Eds.), Coopération et conception, Toulouse: Octarès.

Brézillon P. \& Cavalcanti M. (1997) Modeling and Using Context: Report on the First International and Interdisciplinary Conference CONTEXT-97. Knowledge Engineering Review, 12(4). (to appear)

Brown G. \& Yule G. (1983) The role of context in interpretation. In : Brown G. \& Yule G. Discourse Analysis (chap. 2, pp.27-67), Cambridge University Press. 
Cahour B. (1991) La modélisation de l'interlocuteur : Élaboration du modèle et effets au cours de dialogues de consultation. Thèse de Doctorat de Psychologie Cognitive de l'Université Paris VIII, St-Denis.

Cahour B. \& Paris C. (1991) Role and use of user models. Proc. of the International IJCAI'91 Workshop on "Agent Modelling", Sydney, Australia, August 1991.

Cahour B. \& Karsenty L. (1996) Contextes cognitifs et dysfonctionnements de la communication. Interaction et Cognitions, 1(4), 485-509.

Clark H.H. \& Marshall C.R. (1981) Definite reference and mutual knowledge. In: Joshi A.K., Sag I.A. \& Webber B.L. (Eds.), Elements of Discourse Understanding. Cambridge: Cambridge University Press.

Conklin J. \& Begeman M.L. (1988). gIBIS: A Hypertext Tool for Exploratory Policy Discussion. ACM Transactions on Office Information Systems, 6, 303-331.

Conklin J.E. \& Yakemovic B. (1991) A Process-Oriented Approach to Design Rationale. Human-Computer Interaction, 6, 357-391.

Davies G. (1986) Context effects in episodic memory: A review. Cahiers de Psychologie Cognitive, 6(2), 157-174.

Evans J.St.B.T. (1989) Bias in Human Reasoning. Causes and Consequences. Hillsdale, NJ: LEA.

Flahault F. (1982) Sur le rôle des représentations supposées partagées dans la communication. Connexions, 38, 31-37.

Geslin Ph. (1997) La mer, la terre, et le palétuvier : Ethnologie et transfert de techniques. Thèse de doctorat, Ecole des Hautes Etudes en Sciences Sociales, Paris.

Gibbs R.W. \& Mueller R. (1990) Conversation as Coordinated, Cooperative Interaction. In: Robertson S., Zachary W. \& Black J.B.(Eds.). Cognition, Computing and Cooperation. Norwood NJ: Ablex.

Giboin A. (1997) "Mais de quels symboles tu me parles ?" Les interférences référentielles dans les dialogues coopératifs à distance. In : Falzon P. \& Karsenty L. (Eds.) Rapport final du projet "Dialogue et Coopération" (p. 151-170), Juin 1997, CNAM, Paris.

Gibson J.J. (1979) The ecological approach to visual perception. Boston, MA: Houghton Mifflin.

Grice H.P. (1975). Logic and Conversation. In: Cole P. \& Morgan J.L. (Eds.) Syntax and Semantics, vol.3, N.Y.: Academic Press.

Haviland S.E. \& Clark H.H. (1974) What's New ? Acquiring New Information as a Process in Comprehension. Journal of Verbal Learning and Verbal Behavior, 13, 512-521.

Heath C.C. \& Luff P. (1994) Activité distribuée et organisation de l'interaction. Sociologie du Travail, 4, 523-545.

Heath C.C., Luff P. \& Nicholls G.M. (1995) The Collaborative Production of the Document: Context, Genre and the Borderline in Design. Proceedings of the International Conf. on the Design of Cooperative Systems (COOP'95), pp. 203-218, Juan-Les-Pins, France, 25-27 Janvier.

Hoffman R. R. (1986) Context and Contextualism in the Psychology of Learning. Cahiers de Psychologie Cognitive, 6(2), 215-232. 
Hughes J.A., Randall D. \& Shapiro D. (1992) Faltering from ethnography to design. In Proceedings of the ACM Conf. on Computer-Supported Cooperative Work, pp. 115-122, Toronto, Canada.

Hutchins E. (1990) The technology of team navigation. In: Galegher J., Kraut R.E. \& Egido C. (Eds.) Intellectual teamwork - Social and technological foundations for cooperative work. Hillsdale, NJ: LEA.

Hutchins E. (1995). How a Cockpit Remembers its Speeds. Cognitive Science, 19, 265-288.

Karsenty L. (1995) La production de descriptions d'objets dans un dialogue orienté-tâche. In: Vivier J. (Ed.), Psychologie du dialogue homme-machine en langage naturel. Presses Universitaires de Caen.

Karsenty L. (1996a) An Empirical Evaluation of Design Rationale Documents, Proceedings of the ACM Conference on Human Factors in Computing Systems (CHI'96), Vancouver, 14-18 Avril, ACM Press.

Karsenty L. (1996b) Une définition psychologique de l'explication. Intellectica, 2(23), 299317.

Karsenty L. \& Falzon P. (1993) L'analyse des dialogues orientés-tâche: Introduction à des modèles de la communication. In: Six F. \& Vaxevenoglou X. (1993) Les aspects collectifs du travail. Toulouse: Octarès.

Karsenty L., Bigot V. \& De Brito G. (1995) L'utilisation des procédures écrites dans un avion de nouvelle génération, Rapport EURISCO nT-95-023, Nov. 1995.

Karsenty L. \& Brézillon P. (1995) Coopération Homme-Machine et Explication. Le Travail Humain, 58(4), 291-312

Kobsa A. \& Wahlster W. (Eds) (1989), User Models in Dialog Systems, Berlin: SpringerVerlag

Krauss R.M. \& Fussell S.R. (1990) Mutual Knowledge and Communicative Effectiveness. In: Galegher J., Kraut R.E. \& Egido C. (Eds.) Intellectual teamwork. Social and Technological Foundations of Cooperative Work. Hillsdale, NJ: LEA.

Leplat J. (1986) L'analyse du travail. Revue de Psychologie Appliquée, 31(1), 9-27.

Leplat J. \& Hoc J.M. (1983) Tâche et activité dans l'analyse psychologique des situations, Cahiers de psychologie cognitive, 3(1), 49-63.

MacLean A., Young R.M., Bellotti V.M.E. \& Moran T.P. (1991) Questions, Options, and Criteria: Elements of Design Space Analysis. Human-Computer Interaction, 6, 201-250.

Pavard, B. (1983) Traitement perceptif des mots affixés : mise en évidence d'un contrôle cognitif. L'Année Psychologique, 33, 443-464.

Pavard B., Benchekroun H. \& Salembier P. (1990) La régulation collective des communications: analyse et modélisation. Actes du congrès Ergo-IA'90, Biarritz, France.

Performances Humaines et Techniques (1997), n56, Dossier sur le télétravail.

Rognin L. \& Pavard B. (1996) Pluri-addressed messages and coordination: Impact of the intercom on the human cooperation. Proceedings of the Second Int. Conf. On the Design of Cooperative Systems, June 12-14, Juan-Les-Pins, France, Editions INRIA SophiaAntipolis.

Sacks H., Schegloff E.A. \& Jefferson G. (1974) A Simplest Systematics for the Organization of Turn-Taking for Conversation. Langage, 50, 696-735. 
Sadek D. (1996) Dialogue et coopération. In: de Terssac G. \& Friedberg E. (Eds.) Coopération et Conception. Toulouse: Octarès.

Salembier P. (1993) Le diagnostic cognitif dans les interactions tutorielles. Thèse de Doctorat du CNAM, Paris, Avril.

Salembier, P. (1996) Cognition(s) : Située, Distribuée, Socialement Partagée, etc,... Rapport interne GRIC, ARAMIIHS.

Sauvagnac C. \& Falzon P. (1996) Collaboration and Underlying Issues or the Surprises of Cooperative Dialogues, Computer Supported Cooperative Work, 5(2-3), 251-266.

Schank R.C. (1986). Explanation Patterns. Understanding Mechanically and Creatively. Hillsdale, NJ : LEA.

Schmidt K. (1994) Cooperative work and its articulation: Requirements for computer support. Le Travail Humain, 57(4), 345-366.

Schmidt K. \& Bannon L. (1992) Taking CSCW seriously. Computer Supported Cooperative Work (CSCW), 1(1-2),pp. 7-40.

Schmidt K. \& Simone C. (1995) Mechanims of Interaction: an approach to CSCW Systems Design. Proceedings of the Int. Workshop on the Design of Cooperative Systems, Antibes, 25-27 Janvier, Editions INRIA Sophia-Antipolis.

Ségui J. (1986) The role of context in language processing: When and How ? Cahiers de Psychologie Cognitive, 6(2), 175-186.

Smith N.V. (Ed.) (1982) Mutual Knowledge. London: Academic Press.

Sperber D. \& Wilson D. (1986) Relevance. Communication \& Cognition. Blackwell (trad. fr.: La Pertinence. Communication et Cognition. Les Editions de Minuit. 1989).

Suchman, L. (1987) Plans and situated actions: the problem of human machine communication. New York: Cambridge University Press.

Tang J.C. (1991). Findings from observational studies of collaborative work. Int. Journal of Man-Machine Studies, 34, 143-160.

Terssac G. de \& Chabaud C. (1990) Référentiel opératif commun et fiabilité. In: Leplat J. \& de Terssac G. (Eds.) Les facteurs humains de la fiabilité dans les systèmes complexes. Toulouse : Octarès.

Tiberghien G. (1985) Mais où sont les stimulus d'antan ? Psychologie Française, 30(2), 177183.

Winograd T. \& Florès F. (1987) Understanding computers and cognition, Addison Wesley.

Wisner, A. (1997) Anthropotechnologie : vers un monde industriel pluri-centrique. Toulouse : Octarès.

Zhang J. \& Norman D.A. (1994). Representations in Distributed Cognitive Tasks. Cognitive Science, 18, 87-122.

Zorola-Villarreal, R., Pavard, B. \& Bastide, R. (1995) SIM-COOP: A tool to analyse and predict cooperation in complex environments. A Case Study : The introduction of a datalink between controllers and pilots. In: 5th International Conference on HumanMachine Interaction and Artificial Intelligence in Aerospace, IHM-AI-AS 95, Toulouse, France. 